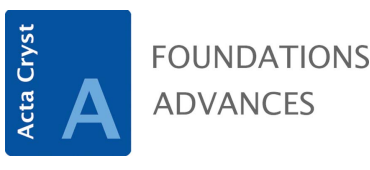

ISSN 2053-2733
Keywords: book review; crystallography; computer applications

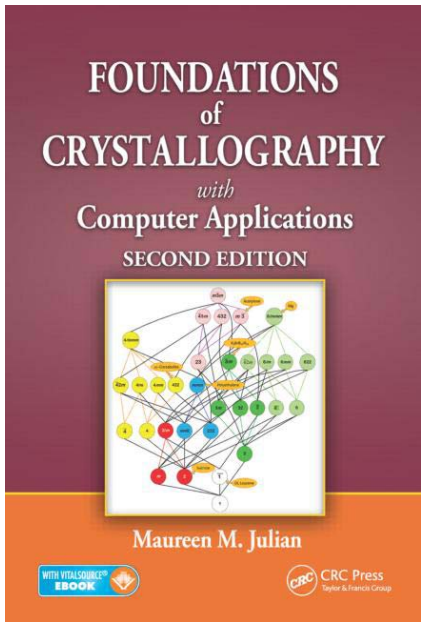

(C) 2016 International Union of Crystallography

\section{Foundations of Crystallography with Computer Applications. Second edition. By Maureen Julian. CRC Press, 2015. Pp. 680. Price USD 84.96 (book and e-book), USD 69.97 (e-book). ISBN 978-1-4665-5291-3.}

\author{
Ulrich Müller* \\ Fachbereich Chemie, Philipps-Universität, D-35032 Marburg, Germany. ${ }^{*}$ Correspondence e-mail: \\ mueller@chemie.uni-marburg.de
}

The aim of this book is to describe the symmetry tools used to interpret crystal structures. The style of the book is striking: lengthy front matter ( $74 \mathrm{pp}$.), including a superfluous 28 pages of lists of tables and figures, lengthy chapter introductions with exhaustive lists of contents and 'chapter objectives', ample space to explain most simple ideas and verbose explanations for simple calculations while being terse when things become more complicated. The text is well understandable for beginners with no previous knowledge, with a clear didactic presentation. Numerous end-of-chapter exercises offer the possibility to apply the learned material; solutions to the problems are not offered either in the book or at the resources for the student on the book's website.

The first sentence of the Introduction reads 'Crystallography is the science of finding the locations of atoms in crystals'. This definition is an inadmissible abridgement of what crystallography really is, what is even evident from the subjects dealt with in the book.

The first five chapters (286 pp.) are devoted to fundamental crystallographic notions: lattices, crystallographic calculations like cell transformations and distance calculations using matrix algebra, reciprocal lattice, basics of group theory including subgroups and trees of group-subgroup relations, symmetry operations and their mathematical treatment, point groups and space groups. Worked-out examples amply explain symmetry as well as calculation procedures using numerical values from real crystal structures. Several basic crystallographic notions have not been included in these introductory chapters, but are postponed to Chapters 9 to 15 , where they are explained in the context of some specific example; for example, fourfold screw axes are mentioned only briefly in Chapter 4 , a more detailed explanation following in the context of the crystal structure of cristobalite in Chapter 12.

All ideas are first developed in two dimensions, 'for clarity and simplicity'. At the beginning, this is OK, but repeating this approach again and again in following chapters becomes tiring and is no longer helpful, taking into account that no real crystal occurs in two-dimensional space. Often the symmetry of a crystal structure is first described by stating the plane-group symbols of projections instead of commenting on the real structure in the first place. For example, Fig. 4.10b shows a structure drawn such that the reader rapidly realizes that it is centrosymmetric (in three dimensions) with atoms on two levels, but it is presented as having twofold rotational symmetry, which is only true for the projection but not for the structure; this unnecessarily complicates the description. It can be positively remarked that, generally, the book strictly observes the rules, symbols and standards of the International Tables for Crystallography, but there are some remarkable exceptions. For example, a mirror plane perpendicular to $y$ is termed $m_{x}$ (in two dimensions) and $m_{x z}$ (in three dimensions) instead of $m_{y}$, which is confusing and not in accordance with the standards.

Chapter 6 deals with X-rays and their diffraction, the emphasis being on theoretical relations and calculations. Aside from the generation of X-rays, experimental aspects are only of marginal interest; the Ewald construction to explain diffraction geometrically is missing. Chapter 7 explains the structure factor and the calculation of electron-density 
maps. This is supported by detailed numerical examples. But clearly, crystal structure analysis is not the subject of this book.

In the last seven chapters (192 pp.) seven selected crystal structures are discussed, one for each crystal system. Each structure is presented in an exhaustive way including the crystal data, discussion of the point group and space group, asymmetric unit, reciprocal lattice, $d$ spacings, atomic coordinates, and calculation of structure factors. The maximal subgroups and minimal supergroups of the space group are listed (disregarding conjugate subgroups and the isomorphic subgroups); there is no mention of what they are useful for. Each example is also used as a thematic anchor to deal with some more general topic, like short and full HermannMauguin symbols, chiral crystal structures and close packings of spheres. Among the 65 Sohncke space-group types that can be adopted by chiral crystals (called proper space groups in this book), chiral and achiral space groups are correctly distinguished. In these seven chapters, many paragraphs are repeated literally again and again in every chapter, with changes only of numerical values and a few terms; is this to discourage the reader from reading all of the chapters?

The book's subtitle 'Computer applications' refers to computer programming instructions to perform a number of simple crystallographic calculations. That is a nice feature, especially for those who have no programming experience; it is restricted to the computer programming language MATLAB.
A useful glossary gives explanations of terms and an appendix lists the symmetry operations, coordinates etc. for the two- and three-dimensional point groups.

The quality of the artwork mainly is excellent. However, some figures of crystal structures are overloaded such that hardly anything can be recognized. Occasionally, a graphical representation is not in accordance with alleged symmetry, like the trigonal unit cell in Fig. 4.42 having a $108^{\circ}$ angle. $2_{1}$ axes are depicted as an ellipse with tails (fonts for graphical symmetry symbols are available at http://www.iucr.org). The letter B depicted on p. 161 to explain mirror symmetry does not have this symmetry.

There are very few misprints, like the 'tetrahedral crystal system' in Table 12.1. The Greek letter $v$ cannot be distinguished from Latin $v$. There are a number of inaccuracies: 'identical' when equal or symmetry-equivalent is meant; 'the reciprocal lattice is a physical property of the crystal' (rather it is an abstract mathematical construction); $\ln \left(V, \AA^{3}\right)$ instead of $\ln \left(V / \AA^{3}\right)$; no distinction between space group and space-group type. Abbreviations like HMB, AA etc. are a user-unfriendly nuisance.

To summarize: generally, this is a wonderful book for beginners with no basic knowledge about crystals, even apt for people who struggle with some of the concepts in crystallography. It is not a book for those who want to go beyond the basics or who are interested in experimental aspects of crystallography. 